\title{
Nachweis myokardialer Ischämien \\ Noch hat das Belastungs-EKG nicht ausgedient!
}

\author{
H.-W.M. Breuer \\ Abteilung für Innere Medizin; Malteser Krankenhaus St. Carolus, Görlitz \\ (Chefarzt Prof. Dr. H.-W. M. Breuer)
}

klinikarzt 2005; $34(8+9): 231-239$

ede Belastungs-EKG-Untersuchung ist auch mit dem Risiko einer potenziellen Schädigung des zu Untersuchenden assoziiert - schätzungsweise erleidet bei 2500 Untersuchungen ein Patient entweder einen Myokardinfarkt oder verstirbt (11). Daher muss grundsätzlich eine eindeutige Indikation gegeben sein. Ein in der Reanimation erfahrener Arzt muss die Belastung überwachen, und es muss eine Reanimationsausrüstung (in erster Linie Defibrillator, Intubationsbesteck und Notfallmedikamente) vorhanden sein. Günstig ist zudem, wenn die Möglichkeit einer Sauerstoffinsufflation über eine Mund- bzw. Nasenmaske gegeben ist, insbesondere bei Patienten mit belastungsinduzierter Hypoxämie.

Die häufigste Indikation zur Durchführung eines Belastungs-EKGs ist der Nachweis einer myokardialen Ischämie. Andere wichtige Einsatzbereiche sind der Nachweis belastungsinduzierter Arrhythmien, die sportoder arbeitsmedizinische Leistungsbeurteilung und die Einteilung in Rehabilitationsprogramme. Absolute Kontraindikationen sind in Anlehnung an die ACC/AHA-Leitlinien (11):

- akuter Myokardinfarkt (vor zwei Tagen)

- akutes koronares Syndrom

- symptomatische unkontrollierte Arrhythmien

Obwohl mit der Koronarangiografie, der Computertomografie (CT) oder der Magnetresonanztomografie (MRT) immer mehr Möglichkeiten bestehen, koronare Stenosen direkt nachzuweisen, oder auch die Auswirkungen einer koronaren Ischämie mittels Spect, Stressechokardiografie und Cardio-MR beeindruckend dargestellt werden können, bleibt das Belastungs-EKG neben der Anamnese immer noch die in der Regel alleine ausreichende Basisdiagnostik zum Nachweis einer myokardialen Ischämie. Um auch unter ökonomischen Aspekten eine akzeptable Sensitivität und Spezifität des Belastungs-EKGs im Kontext der konkurrierenden Verfahren $z u$ erzielen, sind einige grundlegende Voraussetzungen zu beachten. Im Jahr 2002 publizierten das American College of Cardiology (ACC) und die American Heart Association (AHA) (11) Richtlinien zur Durchführung von Belastungsuntersuchungen. Im Wesentlichen bezieht sich der vorliegende Artikel auf diese Empfehlungen, er berücksichtigt dabei jedoch auch zusätzliche neue Erkenntnisse.

- symptomatische schwere Aortenstenose

- symptomatische Herzinsuffizienz

- akute Lungenembolie

- akute Myokarditis oder Perikarditis

- akute Aortendissektion

- jede akute Infektion oder sonstige floride Erkrankung.

Unter klinischen Bedingungen häufig übersehene relative Kontraindikationen sind Elektrolytverschiebungen, zum Beispiel als Folge einer neu eingeleiteten Diuretikatherapie.

In Europa wird die Fahrradergometrie - entweder im Sitzen, Liegen oder auch in einer halb liegenden Position - und in den USA die Laufbandergometrie bevorzugt. Die sit- zende Position ist für sich in gutem Allgemeinzustand befindliche $\mathrm{Pa}-$ tienten aufgrund der ihnen vom Fahrrad fahren gewohnten Position am günstigsten.

Bei liegendem Patienten ist das EKG weniger anfällig für Artefakte. Zudem bieten sich im Liegen bessere Bedingungen für eine potenziell notwendige Reanimation. Ein weiterer Vorteil ist die Vermeidung der Gefährdung durch einen Sturz bei Synkope oder einer Orthostase. Von Nachteil bei der Fahrradergometrie im Liegen ist jedoch eine höhere Volumenbelastung durch den stärkeren venösen Rückfluss (erhöhtes Preload). Damit ist die halbliegende Position ein sinnvoller Kompromiss zwischen beiden Belastungsformen.

Prinzipiell ist die Belastung auf dem Laufband die Belastungsform, 
die der Patient am ehesten gewohnt ist, und muss daher aufgrund von Kraftlosigkeit im Oberschenkel seltener abgebrochen werden als die Fahrradergometrie. In der Regel ist aufgrund des verstärkten Muskeleinsatzes die maximale Sauerstoffaufnahme („Bruttokriterium der kardiopulmonalen Belastung“) um $10 \%$ höher als bei der Fahrradergometrie.

In den USA wird für das Laufband am häufigsten das Bruce-Protokoll mit Belastungssteigerungen in dreiminütigem Abstand durch Veränderung der Laufbandgeschwindigkeit und gleichzeitiger Änderung des Neigungswinkels eingesetzt. Das übliche Belastungsprotokoll bei der Fahrradergometrie beinhaltet eine Steigerung der Belastung um 25 Watt im Abstand von zwei Minuten. In der Regel beginnt die Belastung mit 50 Watt; bei untrainierten oder schmächtigen Patienten kann sie auch bei 25 Watt starten. Bei trainierten Patienten ist ein Belastungsbeginn mit 75 bzw. 100 Watt $\mathrm{zu}$ bevorzugen. Grundsätzlich sollte die Belastung auf eine Zeit von etwa zwölf Minuten limitiert sein, da sonst Faktoren wie mentale oder physische Ermüdung den Belastungsabbruch beeinflussen (4).

Bei Patienten mit Herzinsuffizienz bevorzuge ich persönlich insbesondere bei der Spiroergometrie - die Rampenbelastung mit einer Ausgangsbelastung von 15-25 Watt und einer Steigerung von 10 Watt pro Minute, um eine Gesamtbelastungsdauer von acht bis zwölf Minuten zu erzielen. Bei der Rampenbelastung entfallen die abrupten Belastungssteigerungen, welche die Patienten häufig schlecht tolerieren und daher oft zum vorzeitigen Belastungsabbruch führen.

\section{Prozedere unmittelbar vor der Belastung}

Vor der Belastungsuntersuchung muss sich der Untersucher ein Bild über den aktuellen Zustand des Patienten verschaffen. Ich selbst belaste keinen Patienten, den ich nicht unmittelbar vor der Belastung kardial und pulmonal auskultiert habe. Danach ist dem Patienten der Belastungsablauf zu erläutern. Er ist insbesondere darüber zu informieren, dass es bei drehzahlunabhängigen Ergometern nur erforderlich ist, eine Mindestdrehzahl einzuhalten. Erfahrungsgemäß bevorzugen Trainierte aufgrund eines besseren Kraftwirkungsgrades wesentlich höhere Drehzahlen als Untrainierte. Zudem muss der Patient darauf hingewiesen werden, sich bei Auftreten von Schmerzen, Luftnot oder ähnlichem zu melden und nicht sofort selbstständig die Belastung zu beenden.

Unmittelbar vor der Belastung muss ein Zwölf-Kanal-Ruhe-EKG geschrieben werden, welches der untersuchende Arzt hinsichtlich möglicher Kontraindikationen zur Belastung (dies wäre auch ein technisch insuffizientes „verzittertes“ EKG) auswertet. Zudem sollte in der für die Belastung gewählten Position der Blutdruck bestimmt werden. Einen eindeutigen Grenzwert für Blutdruckwerte, oberhalb derer eine Belastung nicht durchgeführt werden sollte, gibt es nicht. Die ACC/AHALeitlinien schlagen eine Grenze von $200 \mathrm{mmHg}$ systolisch und/oder $110 \mathrm{mmHg}$ diastolisch als relative Kontraindikation vor.

\section{Prozedere während der Belastung}

Während der Belastung sollte der EKG-Verlauf kontinuierlich an einem Monitor beobachtet werden, um Herzrhythmusstörungen recht-

\begin{tabular}{lll} 
Tab. 1 & \multicolumn{2}{l}{$\begin{array}{l}\text { Bedeutung einer individuellen Ausbelastung für die diagnostische } \\
\text { Relevanz eines Belastungs-EKGs }\end{array}$} \\
& $\begin{array}{l}\text { St. Carolus Krankenhaus } \\
\mathbf{1 9 9 6 - 1 9 9 8}\end{array}$ & $\begin{array}{l}\text { Herzinfarktregister } \\
\mathbf{1 9 9 6 - 1 9 9 8}\end{array}$ \\
\hline durchgeführt (\%) & 58 & 33 \\
\hline maximale Belastung (Watt) & 125 & 75 \\
\hline neu aufgetretene Angina pectoris (\%) & 39 & 19 \\
\hline pathologische ST-Senkung (\%) & 48 & 22 \\
\hline
\end{tabular}

zeitig erkennen zu können. Das EKG wird mit einem Papiervorschub von 5-10 $\mathrm{mm} / \mathrm{s}$ geschrieben - falls es nicht durch das EKG-System kontinuierlich gespeichert und retrospektiv gezielt ausgedruckt werden kann. Am Ende einer jeden Belastungsminute oder vor einer neuen Belastungsstufe erfolgt ein Ausdruck mit 25 bzw. $50 \mathrm{~mm} / \mathrm{s}$ Vorschub. Ebenfalls muss vor jeder Belastungssteigerung automatisiert oder manuell der Blutdruck bestimmt werden. Bei nicht plausiblen Messwerten oder einem Abfall des systolischen Blutdrucks trotz weiterer Belastungssteigerung sollte unverzüglich eine Kontrollmessung durchgeführt werden.

Die ACC/AHA-Leitlinien definieren folgende absolute Indikationen zum Belastungsabbruch:

- Abfall des systolischen Blutdrucks um mindestens $10 \mathrm{mmHg}$ gegenüber dem Ausgangsblutdruck trotz eines Anstiegs der Belastung sofern von anderen Zeichen der Ischämie begleitet

- eindeutige Angina pectoris

- zunehmende zerebrale Symptomatik (z.B. Ataxie, Verwirrtheit, Präsynkope)

- Zeichen verminderter peripherer Perfusion (Zyanose oder Blässe)

- technische Gründe, die es nicht möglich machen, das EKG oder den systolischen Blutdruck ausreichend auszuwerten

- der Wunsch des Untersuchten, die Belastung zu beenden (hier sollte der Untersuchende unbedingt verbal intervenieren, wenn keine anderen Indikationen zum Belastungsabbruch vorliegen)

- anhaltende ventrikuläre Tachykardie

- $\quad$ ST-Elevation um mindestens 0,1 $\mathrm{mV}$ in Ableitungen ohne pathologische Q-Wellen (nicht aVR oder V1).

Relative Indikationen zum Belastungsabbruch:

- Abfall des systolischen Blutdrucks um mindestens $10 \mathrm{mmHg}$ gegenüber dem Ausgangsblutdruck trotz eines Anstiegs der Belastung ohne andere Zeichen der Ischämie 
- ST- oder QRS-Veränderungen wie horizontale oder deszendierende ST-Senkung ( $>0,2 \mathrm{mV}$ ) oder ausgeprägter Lagetypwechsel

- Arrhythmien anderer Art als anhaltende ventrikuläre Tachykardien, einschließlich multifokaler ventrikulärer Extrasystolen, Triplets, supraventrikuläre Tachykardien, Blockierungen oder Vorhofflimmern

- Erschöpfung, Luftnot, Giemen, Beinkrämpfe oder Claudicatio

- Entwicklung eines Schenkelblockbildes oder intraventrikuläre Leitungsverzögerung, die nicht von einer ventrikulären Tachykardie zu unterscheiden sind

- zunehmende Angina pectoris

- Hypertonie $(250 \mathrm{mmHg}$ systolisch und/oder $115 \mathrm{mmHg}$ diastolisch).

Das Auftreten einer Angina pectoris ist für die Interpretation eines Belastungs-EKGs von entscheidender Bedeutung. Sprechen die Patienten prompt auf ein schnell wirksames $\mathrm{Ni}$ tropräparat an, weist dies darauf hin, dass tatsächlich eine kardial bedingte Stenokardie besteht. Die wichtigsten EKG-Kriterien für eine belastungsinduzierte Ischämie sind die ST-Streckensenkung oder -hebung. Positive Ischämiehinweise sind eine horizontale oder deszendierende ST-Streckensenkung von mindestens $0,1 \mathrm{mV}$ mindestens 60-80 ms nach dem Ende des QRS-Komplexes (J-Punkt).

In der Literatur immer wieder angegebene Grenzen für den $\mathrm{Ab}$ bruch eines Belastungs-EKGs (z.B. Herzfrequenz 200/min minus Alter oder 220/min minus Alter bzw. erzielte Leistung $>75 \%$ des Solls) sind arbiträr und haben nichts mit einer individuellen Ausbelastung zu tun. Diese Grenzen helfen jedoch die Belastung als submaximal oder maximal einzuordnen. Eine Belastung, bei der die individuelle Ausbelastung gekennzeichnet durch Erschöpfung oder objektive Abbruchkriterien nicht erreicht wird, ist in der Regel mehr Belästigung als Belastung.

\section{Prozedere nach \\ der Belastung}

Um orthostatische oder vagale Dysregulationen $\mathrm{zu}$ vermeiden, macht es Sinn, den Patienten noch einige Zeit im Leerlauf treten zu lassen, sofern kein absolutes Kriterium zum Belastungsabbruch besteht. Eine Nachbeobachtung mit kontinuierlicher Monitorüberwachung und EKG-Ausdruck bzw. -Speicherung erfolgt in der Regel noch vier bis sechs Minuten nach dem Ende der Belastung, mindestens jedoch so lange, bis eventuell belastungsinduzierte EKG-Veränderungen - Reversibilität vorausgesetzt - zurückgebildet sind oder eine Intervention (z.B. Thrombolyse oder Applikation von Antiarrhythmika) eingeleitet wurde.

\section{Kriterien eines pathologi- schen Belastungs-EKGs ST-Senkung}

Das wichtigste Kriterium zur Beurteilung einer myokardialen Ischämie ist der ST-Streckenverlauf. Je ausgeprägter die ST-Streckensenkung ist, umso höher ist die Spezifität für das tatsächliche Vorliegen einer kardialen Ischämie. In der Regel liegt die Sensitivität für den Nachweis einer myokardialen Ischämie etwas niedriger als die Spezifität, die laut Metaanalysen $72 \%$ beträgt (11). Während der Belastung sollen nach Möglichkeit sechs, mindestens jedoch drei Brustwandableitungen kontinuierlich erfasst werden. Die größte diagnostische Sensitivität besitzt VS. Inwieweit zusätzlich rechtspräkordiale Ableitungen von diagnostischer Relevanz sind, ist zurzeit ungeklärt.

Prinzipiell ist davon auszugehen, dass eine computerisierte Analyse des ST-Streckenverlaufs aufgrund der Mittelung mehrerer EKG-Komplexe exaktere Messwerte liefert als eine rein visuelle Analyse. Ob mithilfe dieses exakten Messwertes eine höhere diagnostische Ausbeute verglichen mit einem erfahrenen Untersucher erreichbar ist und inwieweit Fehlbefundungen aufgrund von Artefakten, zu starker Filterung oder Ähnlichem auftreten, ist derzeit nicht klar. Im Sinne der Ausbildung der Assistenten halte ich in Kliniken eine computergestützte Analyse der Belastungs-EKGs für nicht sinnvoll. Auch der Faktor Schnelligkeit sollte keine Rolle spielen, da ein erfahrener Untersucher für die Interpretation eines BelastungsEKGs weniger als eine Minute benötigt.

Eine deszendierende ST-Streckensenkung ist ein besserer Prädiktor für das Vorliegen einer myokardialen Ischämie als eine horizontale Senkung. Beide sind jedoch von höherer diagnostischer Relevanz als eine aszendierende ST-Senkung. Mit einem Anstieg von weniger als $1 \mathrm{mV} / \mathrm{s}$ (zur Erfassung dieses Kriteriums ist allerdings eine computergestützte Vermessung erforderlich) hat Letztere eine höhere diagnostische Vorhersagewahrscheinlichkeit für eine Ischämie als ein schneller ST-Streckenanstieg.

Aszendiert die ST-Strecke, gilt eine „Rest"-Senkung von $0,15 \mathrm{mV}$ $80 \mathrm{~ms}$ nach dem J-Punkt als pathologisch. Geringgradige J-Punktsenkungen von weniger als $0,15 \mathrm{mV}$ finden sich auch bei Gesunden und sollten bei aszendierendem ST-Verlauf $(>1$ $\mathrm{mV} / \mathrm{s}$ ) als normal angesehen werden (19). Um Fehlinterpretationen von ST-Streckenveränderungen zu vermeiden, sollten mindestens drei aufeinander folgende Ableitungen die gleichen Kriterien aufweisen (21).

\section{ST-Elevation}

Die ST-Elevation in einer anderen Ableitung als aVR oder V1 und nicht in Ableitungen mit pathologischen QZacken ist extrem selten und verweist auf eine transmurale Ischämie. Ihre Ursachen sind Spasmen oder kritische Ischämien. Im Gegensatz zur ST-Streckensenkung, die keine spezifische koronare Läsion anzeigt, ist eine ST-Elevation in Ableitung V2-V4 typisch für eine Stenose in der linken Koronararterie. Laterale Ableitungen deuten auf den Ramus circumflexus bzw. Diagonaläste hin und die Ableitungen II, III und aVF auf die rechte Koronararterie.

\section{R-Zacken}

Die R-Zackenamplitude nimmt üblicherweise bis zu einer Herzfrequenz von 130/min zu und reduziert sich dann bis zur maximalen Belastung. Belastungsinduzierte Veränderungen in der R-Amplitude haben keinen unabhängigen prädiktiven Wert. Sie sind jedoch häufig mit 


\section{Tab. 2 Vortestwahrscheinlichkeit für eine koronare Herzkrankheit bei symptomatischen Patienten}

\begin{tabular}{lllllll} 
Alter & \multicolumn{2}{c}{ nichtanginöse Brustschmerzen } & \multicolumn{2}{c}{ atypische Angina pectoris } & \multicolumn{2}{c}{ typische Angina pectoris } \\
\hline (Jahre) & Männer & Frauen & Männer & Frauen & Männer & Frauen \\
\hline $30-39$ & $4 \%$ & $2 \%$ & $34 \%$ & $12 \%$ & $76 \%$ & $26 \%$ \\
\hline $40-49$ & $13 \%$ & $3 \%$ & $51 \%$ & $22 \%$ & $87 \%$ & $55 \%$ \\
\hline $50-59$ & $20 \%$ & $7 \%$ & $65 \%$ & $31 \%$ & $93 \%$ & $73 \%$ \\
\hline $60-69$ & $27 \%$ & $14 \%$ & $72 \%$ & $51 \%$ & $94 \%$ & $86 \%$ \\
\hline
\end{tabular}

dem Vorliegen einer koronaren Herzkrankheit assoziiert, da hier die Belastung in der Regel vor der erst später zu erwartenden R-Zackenreduktion beendet wird.

\section{ST-Herzfrequenz-Adjustierung}

Der ST-Herzfrequenzindex ist eine Anpassung der ST-Streckensenkung an die erreichte maximale Herzfrequenz. Studien mit angiografischer Kontrolle zeigten keine Überlegenheit des ST-Herzfrequenzindex oder des entsprechenden Anstiegs dieses Parameters zum Zeitpunkt des Belastungsabbruchs.

\section{T-Welle}

Das Aufrichten einer negativen T-Welle während der Belastung (TInversion) hat in der Regel keine diagnostische Bedeutung (19). Nur wenn begleitend eine Angina pectoris vorliegt, kann sie auf eine myokardiale Ischämie hinweisen.

\section{Zeit bis zum Belastungsabbruch}

Die Zeiten bis zum Belastungsabbruch, dem Auftreten einer signifikanten Streckenänderung oder einer Angina pectoris sind für die klinische Routine nicht von Bedeutung. Sie sind jedoch relevante Größen in der Überprüfung der Wirksamkeit therapeutischer Interventionen und in der Arzneimittelentwicklung.

\section{Rhythmusstörungen oder Links-} schenkelblock

Belastungsinduzierte Arrhythmien lassen sich weder einer myokardialen Ischämie noch einer sonstigen kardialen Funktionsstörung zuordnen. Ebenso hat das Verschwinden von Extrasystolen während der Belastung keine differenzialdiagnostische Bedeutung. Hingegen haben in der Nachbelastungsphase auftretende Kammerektopien einen höheren prädiktiven Wert für eine ge- steigerte Mortalität als während der Belastung beobachtete Arrhythmien (10). Das Auftreten eines frequenzabhängigen Linksschenkelblocks bei Frequenzen unter 125/min mit anamnestischer Angina weist auf eine koronare Herzkrankheit hin, bei höheren Herzfrequenzen ist die Spezifität geringer (9).

\section{Spezielle Patientengruppen Digitalis}

Unter einer Behandlung mit Digitalis treten in 25-40\% der Fälle auch bei Gesunden ST-Streckenveränderungen auf. Studien, in denen Patienten Digitalis einnahmen, zeigten eine mittlere Sensitivität des Belastungs-EKGs von 68\% und eine mittlere Spezifität von $74 \%$. War Digitalis ein Ausschlusskriterium, lag die Sensitivität des Belastungs-EKGs bei $72 \%$ und die Spezifität bei $69 \%$ (11).

Da eine Digitalistherapie auch bei offensichtlich Gesunden unter Belastung zu einer signifikanten STStreckensenkung führen kann, wird ein Absetzen der Medikation zirka zwei Wochen vor dem EKG empfohlen. Wir führen in der Regel auch Belastungs-EKG-Untersuchungen unter Digitalis durch und stellen hier, abgesehen von Untersuchungen bei Diabetikern, die klinische Symptomatik als Hauptkriterium der Interpretation des Belastungs-EKGs in den Vordergrund. ST-Streckensenkungen von mehr als $0,2 \mathrm{mV}$ scheinen auch unter Digitalis eine hohe Spezifität für das Vorliegen einer Ischämie zu haben (9).

\section{Vorbestehende ST-Strecken- senkung}

ST-Streckensenkungen in Ruhe gelten als Marker für das Auftreten zukünftiger kardialer Ereignisse bei Patienten mit und ohne bekannte koronare Herzkrankheit. Tritt wäh- rend der Belastung zusätzlich eine ST-Streckensenkung von mindestens $0,2 \mathrm{mV}$ oder eine deszendierende ST-Streckensenkung von mehr als $0,1 \mathrm{mV}$ in der Nachbelastungsphase auf, sind dies Indikatoren für das Vorliegen einer myokardialen Ischämie (Sensitivität 67\%, Spezifität $80 \%)$.

\section{Linksschenkelblock}

Extreme ST-Streckensenkungen bei einem Linksschenkelblock sind nicht im Sinne einer myokardialen Ischämie zu beurteilen. Das Vorliegen eines Linksschenkelblocks bewerten die ACC/AHA-Richtlinien (11) als Klasse-IIB-Indikation (Nützlichkeit ist weniger gut durch Studien oder Expertenmeinung belegt) für die Durchführung eines BelastungsEKGs. Dennoch führen wir auch bei einem Linksschenkelblock Belastungs-EKGs durch, wobei hier ausschließlich die klinische Symptomatik und das Auftreten von Rhythmusstörungen beurteilt werden.

\section{Rechtsschenkelblock}

Belastungsinduzierte ST-Streckensenkungen treten üblicherweise bei einem Rechtsschenkelblock in V1 bis V3 auf und weisen nicht auf eine myokardiale Ischämie hin. ST-Streckensenkungen in V5 und V6 sind genau so $\mathrm{zu}$ interpretieren wie bei fehlendem Rechtsschenkelblock.

Betablocker und antianginöse Therapie

Für Routineuntersuchungen ist es nicht erforderlich, die Therapie mit Betablockern zu beenden. Die Gefahr eines „Rebound-Effekts“ (8) ist bei abruptem Absetzen zu beachten. Unter Betablockern ist aufgrund des verminderten Herzfrequenzanstiegs die diagnostische Sensitivität allerdings geringer als ohne Betablockertherapie, da die Herzfre- 
quenz eine physiologische Variable der koronaren Durchblutung ist (der Herzfrequenzanstieg reduziert primär die Dauer der Diastole, in der im Wesentlichen die koronare Durchblutung erfolgt).

Besteht lediglich ein Verdacht auf das Vorliegen einer koronaren Herzkrankheit und wurde bereits eine Betablockertherapie eingeleitet, setzen wir die Medikation 48 Stunden vor einem geplanten Belastungs-EKG ab, um die diagnostische Sensitivität der Untersuchung zu erhöhen. Muss dagegen die Effektivität der Therapie überprüft werden, behalten wir in unserer Klinik die Betablockertherapie bei. Ähnlich verhalten wir uns bei der Therapie mit anderen Antianginosa wie Kalziumantagonisten und Nitraten.

\section{Frauen}

Die diagnostische Spezifität eines Belastungs-EKGs ist bei Frauen, die jünger als 65 Jahre sind, deutlich niedriger als bei Männern. In der CASS ${ }^{1}$-Studie (15) wiesen zirka 50\% der Frauen mit Angina pectoris, die jünger als 65 Jahre alt waren, normale Koronarangiografien auf. Eine Düsseldorfer Untersuchung an 2500 Frauen und 2500 Männern ergab bei Frauen einen positiven prädiktiven Wert für das Vorliegen einer koronaren Herzkrankheit bei Angina pectoris und gleichzeitig pathologischem Belastungs-EKG von 33\% im Vergleich zu $85 \%$ bei Männern. Ohne begleitende Angina pectoris war der positive prädiktive Wert des Belastungs-EKGs bei Frauen 24\%, bei Männern 68\% (17).

Der nur geringe diagnostische Zugewinn durch Einschluss der klinischen Symptomatik bei Frauen zeigte sich auch in anderen Untersuchungen (16). Unter prognostischen Aspekten ist die Herzfrequenzerholungszeit in den ersten beiden Minuten der Nachbelastungsphasen auch bei Frauen von hoher Relevanz. Normal ist ein Abfall der Herzfrequenz von über $12 /$ min während der ersten Nachbelastungsminute oder von über 22/min während der zweiten Minute $(5,16,18)$.

Aufgrund des Bayes-Theorems ist die niedrigere Prävalenz einer koronaren Herzkrankheit bei Frauen

\section{Kasuistik}

E in 61-jähriger Patient wurde von seiner Hausärztin zum Zweck der Abklärung eines „RauE schens im Brustkorb“ zur pneumonologischen Diagnostik überwiesen. Auch bei näherem Befragen gab der Patient nur ein „rauschendes Missempfinden im Brustkorb“ an. Die Symptomatik habe innerhalb der letzten sechs Monate zugenommen. Der Patient litt nicht an einer typischen Angina pectoris oder subjektiv empfundenen Herzrhythmusstörungen. Gelegentlich träte das Gefühl „nicht einatmen zu können“ auf. Bei weiterer Befragung ergab sich die klassische Symptomatik eines Restless-Legs-Syndroms. Die Mutter des Patienten starb im Alter von 61 Jahren an einem Herzinfarkt, der Vater mit 73 Jahren an einem Prostatakarzinom. Der Patient selbst hatte nie geraucht. Außer einer therapierten Hyperlipidämie bestanden keine wesentlichen Vorerkrankungen.

\section{Klinische Untersuchung}

Strömungsgeräusch über rechter Arteria carotis, funktionelles Systolikum im zweiten Interkostalraum rechts, Blutdruck 160/100 mmHg im Sitzen.

\section{Labor}

LDL 2,8 mmol/l (108 mg/dl), HDL 1,4 mmol/l (54 mg/dl), Triglyzeride 1,6 mmol/l (140 mg/dl) unter $20 \mathrm{mg}$ Atorvastatin.

\section{Farbduplexdiagnostik der Halsgefäße}

Intima-Media-Dicke 1,3 mm, Kalkplaque im rechten Bulbus, keine pathologische Strömungsbeschleunigung.

\section{Elektrokardiogramm (EKG) vor und während der Fahrradergometrie}

Vor der Fahrradergometrie war das EKG normal, Abbruch der Ergometrie bei 125 Watt mit „leichtem Druck im Brustkorb“, Herzfrequenz 166/min und einer J-Punkt-Absenkung um 0,2 $\mathrm{mV}$. Eine horizontal bis träg aszendierende ST-Strecke $(0,7 \mathrm{mV} / \mathrm{s})$ lag vor.

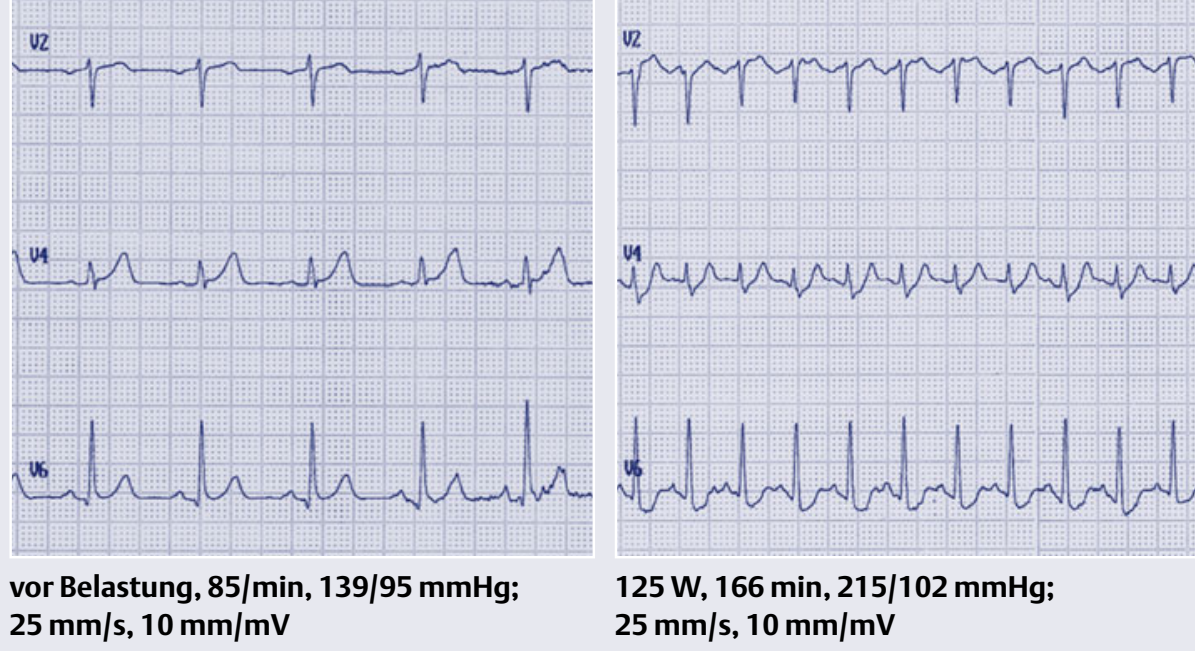

\section{Koronarangiografie}

Im Angiogramm fand sich eine koronare Zwei-Gefäß-Erkrankung mit 90\%er Stenose im proximalen Ramus circumflexus und im ersten Diagonalast. Die Ramus-circumflexus-Stenose wurde mittels PTCA und Stentimplantation behandelt.

\section{Fazit}

Auch bei uncharakteristischer klinischer Symptomatik war das Risikoprofil (männliches Geschlecht, positive Familienanamnese, Hyperlipidämie, arterielle Hypertonie, Karotisarteriosklerose) ausschlaggebend für eine weiterführende Belastungs-EKG-Diagnostik, deren Ergebnis dann zur invasiven Diagnostik und Therapie führte. Nach der Implantation des Stents gab der Patient keine Beschwerden mehr an. 
ein diagnostisches Problem für die richtige Interpretation eines Belastungs-EKGs („geringe Vortestwahrscheinlichkeit"). Dennoch lagen Sensitivität und Spezifität in zahlreichen Analysen nur um weniger als $10 \%$ unter den entsprechenden Werten bei Männern. Insbesondere scheint die eher submaximale Belastung bei Frauen die Wertigkeit des Belastungs-EKGs fälschlich zu reduzieren (11). Als weitere Ursache für die reduzierte Spezifität wird eine gesteigerte Katecholaminausschüttung während Belastung und eine dadurch bedingte Koronarkonstriktion insbesondere während der Menstruation und Präovulation diskutiert (19).

Die Befürchtung einer Fehldiagnose aufgrund falsch positiver Belastungs-EKGs als Basis für weitere Entscheidungen, kann durch eine weiterführende bildgebende Diagnostik wie zum Beispiel eine Stressechokardiografie vermindert werden. Dennoch werden zurzeit bildgebende Belastungsverfahren nicht als initiale Diagnostik für den Nachweis einer myokardialen Ischämie bei Frauen empfohlen (11).

\section{Stellenwert des Belastungs- EKGs 2005}

Aufgrund des positiven prädiktiven Wertes eines Belastungs-EKGs von „nur“ $70 \%$ für den Nachweis einer myokardialen Ischämie, kann ein negatives Belastungs-EKG eine koronare Herzkrankheit nicht ausschließen. Dies gilt insbesondere bei nicht ausreichender Belastung. Bei hohem klinischen Verdacht (typische Angina pectoris) oder hohem familiären Risiko sollte daher die Diagnostik durch weiterführende Belastungsverfahren erweitert werden.

Keine Indikation zur Durchführung eines Belastungs-EKGs besteht bei asymptomatischen Patienten abgesehen von Patienten mit Diabetes oder solchen, die eine Aufnahme regelmäßiger physischer Aktivität (beruflich oder in der Freizeit) planen (11). Während die amerikanischen Empfehlungen bei asymptomatischen Männern, die älter als 45 Jahre sind, und bei Frauen über 55 Jahren eine Belastungsdiagnostik vor regelmäßiger physischer Akti- vität vorschlagen (11), empfiehlt die Deutsche Gesellschaft für Sportmedizin und Prävention ab dem 35. Lebensjahr die ergometrische Belastungs- und Leistungsdiagnostik im Sinne der sportmedizinischen Vorsorgeuntersuchung (14).

Die Bedeutung einer individuellen Ausbelastung für die diagnostische Relevanz eines BelastungsEKGs wird aufgrund des Vergleichs eigener Daten zum Belastungs-EKG am Ende eines stationären Aufenthaltes wegen eines akuten Myokardinfarkts zu den Gesamtkollektivergebnissen im deutschen Myokardinfarktregister deutlich (Tab. 1).

Den Unterschied in der diagnostischen Sensitivität zwischen einer auf $70 \%$ der Sollherzfrequenz limitierten Belastung und einer symptomlimitierten Belastung veranschaulicht eine Studie an 150 konsekutiven Patienten $(6,4 \pm 3,1$ Tage nach Myokardinfarkt, Follow-up von 15 Monaten): $23 \%$ der Patienten hatten bei einer $70 \%$ igen Sollherzfrequenzbelastung eine positive Ergometrie und $40 \%$ bei symptomlimitierter Belastung. Nachfolgend trat bei $32 \%$ der Patienten mit pathologischem Belastungs-EKG, die nach einer "Sollherzfrequenz“ belastet wurden, ein kardiales Ereignis auf und bei $62 \%$ der Patienten mit pathologischem Belastungs-EKG bei symptomlimitierter Belastung (1).

Für die Interpretation eines Belastungs-EKGs ist die Vortestwahrscheinlichkeit (Bayes-Theorem) von Bedeutung. Für einen 20-Jährigen ist die Wahrscheinlichkeit, eine koronare Herzerkrankung aufzuweisen, auch bei einem pathologischen EKGBefund äußerst gering. Wahrscheinlicher sind in diesem Fall Elektrolytverschiebungen, ein Mitralklappenprolaps oder Präexzitationssyndrome. Anders ist dies bei einem 50jährigen Raucher. Bei diesem Patienten ist die Wahrscheinlichkeit für das Vorliegen einer koronaren Herzkrankheit hoch, wenn ein pathologisches Belastungs-EKG vorliegt.

Die Leitlinien der ACC, der AHA, des „American College of Physicians“, der „American Society of Internal Medicine" (ACP-ASIM) und der Deutschen Gesellschaft für Kardiologie (DGK) stellen die Vortestwahr- scheinlichkeit hinsichtlich des Vorliegens einer signifikanten koronaren Herzkrankheit bei symptomatischen Patienten entsprechend verschiedener Altersklassen und dem Geschlecht zusammen (Tab. 2; 6, 12).

Aufgrund der Vortestwahrscheinlichkeit schließt Greenland (13), dass bei Patienten mit niedrigem A-priori-Risiko $(<10 \%$ in zehn Jahren entsprechend den NCEP-Leitlinien [„National Cholesterol Education Program“]) jegliche nichtinvasive Diagnostik wegen ihrer zu geringen Spezifität bezüglich des aufgrund eines positiven Befundes erwachsenden Risikos einer invasiven Diagnostik mit zu erwartender negativer Aussage fragwürdig sei („ungünstiges Nutzen-/Risiko-Verhältnis“). Bei Patienten mit hoher Vortestwahrscheinlichkeit (20\% oder höheres Risiko für ein koronares Ereignis innerhalb von zehn Jahren) kann auch eine negative, nichtinvasive Diagnostik wegen ihrer eingeschränkten Sensitivität eine koronare Herzkrankheit nicht sicher ausschließen. Von besonderer Relevanz ist die nichtinvasive Belastungs-EKG-Diagnostik für jene Patienten, die ein intermediäres Risiko für ein koronares Ereignis zwischen 10 und 20\% in den nächsten zehn Jahren aufweisen.

Um die kardiopulmonale Funktion beurteilen und zwischen kardialer und pulmonaler Limitierung einer Belastung differenzieren zu können, bietet sich die Spiroergometrie an, die per se eine Belastungs-EKG-Diagnostik einschließt (4). Sinnvolle Ergänzungen oder Alternativen für die Lokalisationsdiagnostik einer myokardialen Ischämie sind die Stressechokardiografie oder Myokardszintigrafie. Alternativ ist zur Durchführung einer Koronarangiografie auch eine morphologische Diagnostik mittels CT und MRT möglich.

Es ist zu erwarten, dass die nichtinvasiven bildgebenden Verfahren auch unter Berücksichtigung

\footnotetext{
${ }^{1}$ coronary artery surgery study

2 danish multicenter randomized study of invasive versus conservative treatment in patients with inducible ischemia after thrombolysis in acute myocardial infarction
} 
der möglichen Funktionsdiagnostik (Cardio-MR) einen immer höheren Stellenwert erreichen werden. Dennoch werden sie wohl aufgrund ihrer eingeschränkten Verfügbarkeit und der hohen Kosten das Belastungs-EKG als primäre Untersuchungsmethode zum Nachweis einer myokardialen Ischämie in den nächsten Jahren nicht ablösen.

Das übliche Einsatzgebiet eines Belastungs-EKGs ist die Diagnostik mit einer allerdings geringen Relevanz bei asymptomatischen Patienten mit kardiovaskulärem Risiko. Unter prognostischen Aspekten ist jedoch der Einschluss von Parametern des Belastungs-EKGs einer alleinigen Risikoabschätzung aufgrund konventioneller Risikofaktoren signifikant überlegen. Hierbei repräsentieren die klassischen Risikofaktoren primär das Risiko für die Entwicklung einer Arteriosklerose. Die Belastungsparameter dagegen sind in diesem Patientenkollektiv Indikatoren einer asymptomatischen koronaren Herzkrankheit (7).

Ähnlich wie bei diesen Daten aus Norwegen kommt auch eine Analyse an 3343 Framingham-Teilnehmern zu dem Schluss, dass bei asymptomatischen Männern das Auftreten einer ST-Streckensenkung von mindestens $0,1 \mathrm{mV}$, das Nichterreichen der Zielherzfrequenz oder der altersnormierten Belastung im BruceProtokoll die Prädiktion koronarer Ereignisse über den klassischen Framingham-Score hinaus signifikant verbesserte (2).

Sogar das isolierte Symptom „Atemnot" als Abbruchbefund bei der Fahrradergometrie von gesunden Männern war über 26 Jahre prognostisch assoziiert mit einem 1,86fachen Risiko für einen tödlichen Myokardinfarkt, einem 1,64fach erhöhten allgemeinen Mortalitätsrisiko und einem 3,47fach erhöhten Risiko für eine tödliche pulmonale Erkrankung verglichen mit Männern, die die Belastung aufgrund Erschöpfung beendeten (3).

Die Bedeutung älterer Studien zur Analyse der prognostischen Wertigkeit eines Belastungs-EKGs nach einem Myokardinfarkt wurde in den letzten Jahren zunehmend infrage gestellt, da sich das therapeuti- sche Vorgehen beim akuten Myokardinfarkt relevant geändert hat. In der DANAMI2-2-Studie wurde ein erschöpfungslimitiertes BelastungsEKG bei $79,6 \%$ der Patienten zum Zeitpunkt der Entlassung nach akutem Myokardinfarkt durchgeführt (4-14 Tage, Median: sechs Tage nach Myokardinfarkt). Das mediane Follow-up betrug 3,1 Jahre. Trotz einer Betablockertherapie erreichte über die Hälfte der Patienten mehr als $70 \%$ der maximalen Herzfrequenz (220 - Lebensalter). Die Gesamtbelastungsfähigkeit - ausgedrückt als metabolische Äquivalente: METS = [13.x Watt / Gewicht (kg)+3,5] / 3,5) - war der stärkste Prädiktor für Tod oder erneuten Myokardinfarkt bei den lysierten oder koronarangiografierten Patienten.

Es fand sich ein mehr als dreifacher Unterschied bei den Patienten mit einer Belastungsfähigkeit von über acht und jenen von unter sechs metabolischen Äquivalenten. Auffallend war, dass die ST-Streckensenkung nur bei den lysierten Patienten ein unabhängiger Risikoprädiktor war, jedoch nicht bei den Patienten, die einer perkutanen Koronarintervention (PCI) zugeführt worden waren. Patienten in der PCI-Gruppe mit oder ohne ST-Streckensenkung hatten nahezu die gleichen Ereignisraten $(20)$.

Detection of Myocardial Ischaemia The Exercise ECG is by no Means Redundant

Coronary angiography, computed tomography (CT) and magnetic resonance tomography (MRT) now offer more options for the direct detection of coronary stenosis, and the sequelae of coronary ischaemia can be impressively represented with the aid of SPECT, stress echocardiography and cardiac MR. Nevertheless, the exercise $E C G$, together with the case history, still suffices to enable the reliable basic diagnosis of myocardial ischaemia. In order to ensure acceptable sensitivity and specificity of the exercise ECG - also under economic aspects - within the framework of competing procedures, a number of basic requirements need to be considered. In the year 2002, the American College of Cardiology $(A C C)$ and the
American Heart Association (AHA) (11) issued a guideline update for the performance of exercise testing. For the most part, the present article discusses these recommendations, but also takes into account new facts.

\section{Key Words}

exercise ECG - myocardial ischaemia - right bundle-branch block - left bundle-branch block - ST segment depression - ST segment elevation $T$ wave - $R$ wave

\section{Literatur}

1. Avanindra J, Myers GH, Sapin PM et al. J Am Coll Cardiol 1993; 22: 1816-1820

2. Balady G], Larson MG, Vasan RS et al. Circulation 2004; 110: 1920-1925

3. Bodeguard J, Erikssen G, Bjørnholt JV et al. Europ Heart J 2005; 26: 1394-1401

4. Breuer HWM. Pneumologie 2004; 58: 553-565

5. Cole CR, Blackstone EH, Pashkow FJ et al. New Engl J Med 1999; 341: 1351-1357

6. Dietz R, Rauch B. Z Kardiol 2003; 92: 501-521

7. Erikssen G, Bodegard J, Bjørnholt JV et al. Europ Heart ] 2004; 25: 978-986

8. ESC Expert consensus document on $\beta$ adrenergic receptor blockers. Europ Heart 2004: 25: 1341-1362

9. Fletcher GF, Schlant RC in Hurst's „The Heart“. The exercise test ( $9^{\text {th }}$ edition). The McGraw Hill Company 1998, 519-536

10. Frolkis JP, Pothier CE, Blackstone EH, Lauer MS. N Engl J Med 2003; 348: 781-790

11. Gibbons RJ, Antmann E. www.acc.org

12. Gibbons RJ. J Am Coll Cardiol 1999; 33: 2092-2197

13. Greenland P, Gaziano JM. N Engl J Med 2003; 349: 465-473

14. Jeschke D, Zeilberger K. Dtsch Ärzteb 2004; 101: A789-A798

15. Kennedy H, Killip T, Fischer L et al. Circulation 1977; 56: 756-761

16. Mieres JH, Chair LJS, Arai A et al. Circulation 2005; 111: 682-696

17. Schannwell CM, Schoebel FC, Lazica D et al. Dtsch Med Wschr 2000; 125: 14171423

18. Shetler K, Marcus R, Froelicher VF et al. J Am Coll Cardiol 2001; 38: 1980-1987

19. Trappe H-J, Löllgen H. Z Kardiol 2000; 89: 821-837

20. Valeur N, Clemmensen P, Saunamäki K, Grande P for the DANAMI-2-investigators. Europ Heart | 2005; 26: 119-127

21. Worth H, Breuer H-WM. Pneumologie 1998; 52: 225-231

\section{Anschrift des Verfassers}

Prof. Dr. Hans-Willi M. Breuer, FCCP, FESC

Abteilung für Innere Medizin

Malteser Krankenhaus St. Carolus

Carolusstr. 212

02827 Görlitz 